\title{
Kenneth O. Stanley and Joel Lehman: Why greatness cannot be planned: the myth of the objective
}

Springer, 2015, Softcover, ISBN: 978-3-319-15523-4

\author{
Leonardo Trujillo ${ }^{1}$
}

Published online: 27 October 2015

(C) Springer Science+Business Media New York 2015

"The successful inventor asks where can we get from here rather than how can we get there" (page 97). Kenneth O. Stanley and Joel Lehman provide a bevy of thought provoking insights such as this one throughout Why Greatness Cannot Be Planned-The Myth of the Objective. It is a very nicely written and enjoyable book, aimed at a general readership. It is also surely worthwhile reading for Artificial Intelligence (AI) researchers, particularly for those of us working in genetic programming. Stanley and Lehman use the lessons learned from their own research in AI to provide interesting and plausible hypotheses that resonate at a wider scale.

The introductory quote captures one of the main messages of the book. It forces us to question the very essence of how a search process should be performed when our goal is to discover something great. What is particularly interesting is that the quote seems like perfectly reasonable advice, of the type usually given by a professor or mentor. However, it's main idea is largely overlooked by many that design formal approaches towards problem solving, be it optimization in science and engineering or the shaping of public policy.

The main thesis of the book might be controversial. Stanley and Lehman argue, in many passages quite elegantly, that modern society erroneously clings to what they refer to as the myth of the objective. The idea that every goal worth reaching can be stated as a clear and measurable objective, a guiding beacon. Many of us assume that if we define the correct beacon and use it to infer the most promising search direction then the only thing standing in our path is the required desire and effort. Unfortunately, particularly for truly challenging problems, as Lehman and

Leonardo Trujillo

leonardo.trujillo@tectijuana.edu.mx

1 Departamento de Ingeniería Eléctrica y Electrónica, Posgrado en Ciencias de la Ingeniería, Instituto Tecnológico de Tijuana, Unidad Otay, Av. Alberto Limón Padilla S/N, Mesa de Otay, C.P. 22500 Tijuana, BC, México 
Stanley point out, most beacons are deceptive and will lead us astray. Therefore, they argue, instead of striving for beacons that we cannot see through the fog of ignorance, we should instead take a more subjective approach, guided by the intuitions developed through past experiences, whilst freely exploring the space of possibilities. Stanley and Lehman describe promising new possibilities as stepping stones and the processes that finds and builds upon them as stepping stone collectors, identifying the human ability for invention and biological evolution as two noteworthy examples.

The book is on the short side, with only 141 pages divided into eleven chapters and organized into two parts. The first part encompasses chapters 1 through 9, where the authors present their main arguments. These chapters are intended for a broader audience and do not require formal scientific training. The second part, chapters 10 and 11, is intended for researchers familiar with evolutionary theory or $\mathrm{AI}$ and offers a more detailed analysis of the consequences that the myth of the objective can have in specific academic fields.

Chapter 1 describes how modern society considers objectives to be a prerequisite of any worthwhile endeavor. Stanley and Lehman rely on a variation of Jorge Luis Borges' The Library of Babel, posing discovery and invention as a search process, a thought experiment previously used by Daniel Dennett in his influential book Darwin's Dangerous Idea. Afterwards, through a series of real-world examples, Chapter 2 attempts to show that great achievements can be reached without requiring objectives; unfortunately the authors rely heavily on anecdotal evidence. Chapter 3 describes Stanley's work on Picbreeder, a collaborative website based on interactive evolutionary search. The Picbreeder examples suggest that a system that lacks a single clearly defined goal can still produce impressive designs (in this case pictures), unexpectedly outperforming objective-based search. Stanley and Lehman make an analogy with animal breeding but omit the fact that animal breeders do have objectives, a contradiction they fail to resolve. Chapter 4 is eloquently written, taking the insights provided by Picbreeder and extrapolating them further, arguing that most lofty goals cannot be reached by using objectives because most objectives will prove to be deceptive; a hypothesis that many of us in our field will surely agree with. Their thought experiment of imagining the earth as a huge Petri dish in which a crafty breeder must evolve intelligent beings is particularly attractive and easy to understand. In Chapter 5 they present their novelty search paradigm, a search process developed for evolutionary robotics and their second main source of inspiration in writing this book. Stanley and Lehman differentiate novelty search from random search, describing it as a principled search strategy that orders the search space from the simple to the complex. Chapter 6 builds on the previous chapters, it presents novelty search and the Picbreeder approach as plausible alternatives for design and engineering. Chapters 7 and 8 go a step further, expanding on these ideas and evaluating important social policies. They convincingly argue that efforts aimed at maximizing social indicators will mostly fail and produce negative consequences. Chapter 7 seems to echo the message of many critics of current educational practices, such as Noam Chomsky, stating that diversity and freedom should be the core elements of any education system. In Chapter 8 they present a similar analysis for the way in which science is currently 
prioritized and funded. They defend the view that low risk attitudes toward scientific inquiry are ill-conceived and should be replaced by policies that promote creative and unique research. Chapter 9 concludes the first part of the book with an eloquent adieu to stringent objective-based thought.

The second part of the book provides a more detailed discussion of how objective-free thinking can help recast specific scientific endeavors. In Chapter 10 Stanley and Lehman analyze biological evolution, correctly pointing out that survival and reproduction are constraints imposed by nature and should not be seen as explicit objectives. They present a thought experiment they refer to as Gentle Earth, a planet similar to ours where every individual that ever existed had a chance to procreate without worrying about natural selection. Gentle Earth contains all current species plus a wide range of other lineages that did not survive on Earth. Thus they suggest that natural selection is not responsible for the ability of evolution to adapt and produce surprising new designs. While the arguments merit further inquiry, Stanley and Lehman focus exclusively on the individual level of selection and do not consider how this hypothesis might be reshaped when analyzed at the gene-centric level, as propounded by Richard Dawkins and others. The final chapter discusses the impact that objective-based reasoning has within AI and the development of search algorithms. Stanley and Lehman present an interesting metaanalysis, noting that our community is in fact performing a search over all possible search algorithms, but that we have mostly failed at using this introspection to our advantage; an analysis that the genetic programming community will definitely appreciate.

In closing, I recommend Why Greatness Cannot be Planned. It is definitely unique within the evolutionary computation community. That said, the book does suffer from some notable weaknesses, particularly the limited depth with which some issues are discussed and their reliance on limited amounts of hard data. Nonetheless, I hope that some authors in our field will follow their example and communicate our work to a broader public, in an attempt to help our society and culture find "a path not limited by the shackles of a mandated outcome" (page 91). 